\title{
Charging effects and quantum crossover in granular superconductors
}

\author{
E. Granato \\ LAS, Instituto Nacional de Pesquisas Espaciais, \\ 12.225 São José dos Campos, S.P. Brazil. \\ M.A. Continentino \\ Instituto de Física, Universidade Federal Fluminense, \\ Outeiro de S.J.Batista s/n, Niteroi, 24.020, RJ, Brazil.
}

\begin{abstract}
The effects of the charging energy in the superconducting transition of granular materials or Josephson junction arrays is investigated using a pseudospin one model. Within a mean-field renormalization-group approach, we obtain the phase diagram as a function of temperature and charging energy. In contrast to early treatments, we find no sign of a reentrant transition in agreement with more recent studies. A crossover line is identified in the nonsuperconducting side of the phase diagram and along which we expect to observe anomalies in the transport and thermodynamic properties. We also study a charge ordering phase, which can appear for large nearest neighbor Coulomb interaction, and show that it leads to first-order transitions at low temperatures. We argue that, in the presence of charge ordering, a non monotonic behavior with decreasing temperature is possible with a maximum in the resistance just before entering the superconducting phase.
\end{abstract}

$74.40+\mathrm{k}, 64.60 . \mathrm{Cn}$

Typeset using REVTEX 


\section{INTRODUCTION}

The recent discovery of high temperature superconductors has renewed the interest in granular materials. These systems appear to have an intrinsic "granularity" which is found even in single crystalst. A clear manifestation of this non-uniformity in the scale of the Ginsburg-Landau coherence length is the two-step nature of the transition to the superconducting state 2 . 3 . As temperature is lowered, first the superconducting ordered parameter is developed in each grain at a temperature $T_{c o}$ but because the thermal energy is higher than the Josephson coupling between the grains $E_{J}$, the phases of the order parameter are uncorrelated due to thermal fluctuations. Only at a lower temperature $T_{c} \sim E_{J}$ will phase locking take place leading to long range phase coherence and zero resistivity. Besides its possible relevance for high temperature superconductors granular superconductors have been an active field of research for many years 1 - 16 .

One of the most important issues in the granular superconductors materials is the role of the charging effects on the phase coherence transition. As pointed out by Abeles $\mathbb{A}$, when the grain charging energy $E_{c} \sim e^{2} / d$, where $d$ is the grain diameter and $e$ the electronic charge, is larger than $E_{J}$ long-range phase coherence is destroyed due to zero point fluctuations of the phase of the superconducting order parameter. The onset of phase coherence with increasing intergrain Josephson coupling can then be viewed as a zero-temperature phase transition. Also, of great interest is the resulting phase diagram as a function of temperature and charging energy which can display reentrant transitions to the normal state upon cooling the system to low temperatures. This possibility and its experimental observation have been a matter of much debate-10. More recently, an intriguing effect has been discovered at the onset of superconductivity in granular systems where the resistivity rises steeply, attaining a sharp maximum, before vanishing with decreasing temperature17.

In this paper we study a pseudospin one hamiltonian for granular superconductors which takes into account self-charging effects in the grains, the inter-grain ( short-range ) Coulomb interaction and the Josephson coupling between the phases of the Ginzburg-Landau order 
parameter of the grains. This model has been proposed by de Gennes and studied in some detail specially in relation to the issue of reentrant behavior 10,13 . Within a mean-field renormalization-group approach, we obtain the phase diagram as a function of temperature and charging energy but find no sign of a reentrant transition in agreement with more recent studies. We also study the quantum to classical crossover which takes place in the non-superconducting side of the phase diagram and identify a crossover line along which we expect to observe anomalies in the transport and thermodynamic properties with decreasing temperature. Finally, we study a charge ordering phase, which can appear for large nearest neighbor Coulomb interaction, and show that it leads to first-order transitions at low temperatures. Then we argue that, in the presence of charge ordering, a non monotonic behavior with decreasing temperature is possible with a maximum in the resistance just before entering the superconducting phase, which could in principle be observed experimentally.

\section{PSEUDOSPIN ONE MODEL}

The standard model for granular superconductors, in the absence of disorder and dissipation, consists of a regular array of superconducting grains coupled by Josephson junctions described by the following Hamiltonian

$$
H=2 \sum_{i, j} U_{i j} n_{i} n_{j}-E \sum_{<i j>} \cos \left(\phi_{i}-\phi_{j}\right)
$$

where $U_{i j}$ is the charging energy due to Coulomb interaction and $E>0$ is the Josephson energy. Here $n_{i}$ is the excess of Cooper pairs in the ith grain and $\phi_{i}$ is the phase of the

order parameter. In the pseudospin one approximation[10.18 to the above Hamiltonian one identifies the pair number operator $n_{i}$ with the $z$-component $S_{i}^{z}$ of a spin one operator. The second term in Eq. (1) when expressed in terms of $\exp \left( \pm \phi_{i}\right)$ can then be rewritten as raising and lowering operators $S_{i}^{ \pm}$. If we make the additional approximation of short range Coulomb interaction this results in the following Hamiltonian

$$
H=2 U \sum_{i}\left[S_{i}^{z}\right]^{2}+4 V \sum_{<i, j>} S_{i}^{z} S_{j}^{z}-\frac{E}{4} \sum_{<i, j>}\left(S_{i}^{+} S_{j}^{-}+S_{i}^{-} S_{j}^{+}\right)
$$


The first term describes intragrain Coulomb energy $U>0$ for different charge states. These states, in different grains, are coupled through the short-range nearest neighbor Coulomb interaction $V>0$. The factors 2 and 4 stand for the charge of the excitations which are Cooper pairs. The last term is ultimately responsible for the phase locking of the different grains. It is clear that even at zero temperature this hamiltonian may give rise to phase transitions due, for example, to a competition between the self-charging term $U$ and either the intragrain charge interaction $V$ or the Josephson coupling $E$. In the former case this competition can lead to an instability with the formation of charge dipoles for large $V$. In the latter the competition gives rise to an off-diagonal long range ordered state characterized by the order parameter $\left\langle S^{x}\right\rangle$. The fact that the above hamiltonian takes into account only charge fluctuations of $\Delta n= \pm 1$ is not expected to change the universality class of the zero-temperature superconductor-insulator transition considered here.

\section{MEAN FIELD RENORMALIZATION GROUP}

The mean-field renormalization group has been extensivily applied to a variety of prob-

lems both classical and quantum 19,20, with 21 and without disorder22. This method represents an improvement over the mean-field and the Oguchi pair approximation 9 since fluctuations are included at a higher level. This gives rise for example to critical exponents which assume non-mean-field values2 22 and to a vanishing critical temperature for the two-dimensional Heisemberg model20, in agreement with the well known Mermin and Wagner theorem23. We shall employ this method here to investigate the superconducting transition described by the hamiltonian of Eq. (2). The main reason of doing this is to help settle the issue of the existence (or not) of a reentrant transition at low temperatures 0 - 10 .

As mentionned before for large values of $U$ we expect to find the system in a well defined ( neutral ) charge state and consequently with no phase coherence. The order parameter describing the phase coherent state, expected to occur when the Josephson coupling becomes sufficiently large compared to $U$, is the transverse magnetization $<S^{x}>$. The mean-field 
renormalization group relies on a scaling relation between quantities calculated using two different finite systems (or cells) with appropriate boundary conditions. Within its simplest version, one consider two cells containing one and two spins each with corresponding mean fields $b^{\prime}$ and $b$ acting at the boundaries of the cells. For each cell we need to calculate the derivative of the order parameter $\left\langle S^{x}\right\rangle$ for vanishing mean field. For the spin one model of Eq. (2) we obtain for the one spin cell

$$
\frac{\partial<S^{x}>^{\prime}}{\partial b^{\prime}}=\frac{e}{2} c\left(e^{\frac{2}{t^{\prime}}}-1\right) /\left(2+e^{\frac{2}{t^{\prime}}}\right)
$$

where we have defined the dimensionless parameters $v=V / U, e=E / U$ and $t=k_{B} T / U$ and $c$ is the number of nearest heighbors. Due to the mean-field like character of the approach that we discuss here, we expect the results to be more likely to hold in three dimensions, i.e., $c=6$. For the two spin cell we obtain

$$
\begin{aligned}
\frac{\partial<S^{x}>}{\partial b}= & \frac{e}{4 Z_{o}}(c-1)\left(\frac{-2 e^{\frac{4(-1+v)}{t}}}{2-\frac{e}{2}-4 v}-\frac{2}{e^{\frac{4+e}{2 t}}\left(-2+\frac{e}{2}+4 v\right)}\right. \\
& +\frac{4 e^{\frac{-4+e}{2 t}}\left(16-32 e-5 e^{2}-32 v-24 e v+128 v^{2}\right)}{64+16 e+4 e^{2}+e^{3}+16 e^{2} v-256 v^{2}+64 e v^{2}} \\
& -\frac{4}{e^{\frac{4(1+v)}{t}}\left(2+\frac{e}{2}+4 v\right)} \\
& +\frac{2 e^{\frac{-4+4 v+d}{2 t}}}{(-e+4 v+d)} \\
& \left.\times \frac{(-4-e+4 v-d)^{2}}{\left(\frac{d^{2}}{2}+2 d-2 d v\right)}\right)
\end{aligned}
$$

where

$$
Z_{o}=\frac{2}{e^{\frac{4-e}{2 t}}}+\frac{2}{e^{\frac{4+e}{2 t}}}+e^{\frac{-4(1-v)}{t}}+\frac{2}{e^{\frac{4(1+v)}{t}}}+e^{\frac{-(4-4 v-d)}{2 t}}+e^{\frac{-(4-4 v+d)}{2 t}}
$$

and $d=\sqrt{2} \sqrt{8+e^{2}-16 v+8 v^{2}}$.

We now impose the scaling relation 19 ,

$$
\frac{\partial<S^{x}>^{\prime}}{\partial b^{\prime}}=\frac{\partial<S^{x}>}{\partial b}
$$

where the primed quantities are calculated in the smaller cell. This equation results from the assumption that the order parameter and the mean field scale in the same way and it 
provides recursion relations for the couplings or their ratio $(U / V)$ in terms of the parameters: temperature, the interaction $\mathrm{V}$ and the number of nearest neighbors $c$. The unstable fixed point, as usual in the renormalization group procedure, is associated with the critical point at which the critical exponents are obtained. The phase boundary obtained from this recursion relation, for $V / U=0$ and $V / U \neq 0$, is shown in Fig. 2 together with the result obtained using the Oguchi pair approximation ${ }^{6}$ which can be regarded here as a self consistent solution of a two site cell approximation. As expected, for the same $\mathrm{V}$, the $T=0$ value of the critical ratio, $(E / U)_{c}$, for the appearance of the phase-locked state is larger for the mean field renormalization group calculation then for the Oguchi method due to a better treatment of fluctuations. Notice that as $V$ increases a larger value of $E$ is required to stablish offdiagonal long range order. Also no sign of reentrant behavior is found in our results. We point out that in the calculation above we assumed no charge ordering which, as will be discussed in Sec. 5, only holds for $10 \mathrm{~V} / U<v_{c}$, where $v_{c}=1 / c$.

The critical line close to the zero temperature fixed point in Fig. 1 rises in temperature as:

$$
\left(\frac{E}{U}\right)-\left(\frac{E}{U}\right)_{c} \propto \exp \left(-U / k_{B} T\right)
$$

The exponential dependence is an artifact of the mean-field nature of the renormalization group approach and appears for any dimension. In fact for $d=3$ we would expect to find a power law dependence for the critical line, i.e., $(E / U)-(E / U)_{c} \propto T^{1 / \phi}$ where $\phi=\nu z$ is the crossover exponent. Here, $\nu$ is the correlation-length and $z$ is the dynamical critical exponent. The critical exponents $\nu$ and $z$ are associated with the $T=0$ fixed point at $(E / U)_{c}$. Since this transition is expected to be in the universality class of the $d+1$ classical XY mode1 11,12 we have $z=1$ and $\nu=0.67$ and $1 / 2$ for $d=2$ and 3 respectively. We should point out that our mean field renormalization group calculation yields a lower critical dimension $d_{l}=1.95$ for the finite temperature superconducting instability which is close to the known result for models with continuous symmetry 23 . The existence of a lower critical dimension within the mean field renormalization group shows its significant improved nature compared to the 
usual mean-field methods.

\section{QUANTUM CROSSOVER}

As can be seen from the phase diagram in Fig. 1 for $(E / U)<(E / U)_{c}$ superconductivity in a macroscopic scale is inhibited due to charging effects. We may however expect to find, even in this non-critical part of the phase diagram but sufficiently close to the critical point, signs of the incipient long range superconducting instability. This should occur as anomalies in the transport properties, such as minima in the resistivity or in thermodynamic quantities. Where are such anomalies expected to occur? In a tentative to clarify this point Fazekas et al. 10 and Fazio et al 13 have calculated the transverse and longitudinal correlation function respectively for a pair of spins in the non-critical region. They found in the phase diagram of Fig. 1 a line of extrema for these quantities whic $\mathrm{h}$ intercept the critical line for $(E / U)>(E / U)_{c}$ at a finite temperature. However in their calculations they have completely neglected the effect of fluctuations of the surrounding medium. Also, if this effect is to be associated with a quantum crossover temperature $T^{*}$ one would expect from general scaling arguments, close to the $T=0$ superconductor insulator transiton 24, 1 , that this temperature should approach zero as $\left.T^{*} \sim\left(\frac{E}{U}\right)_{c}-\frac{E}{U}\right)^{z \nu}$.

The fluctuations ignored in the treatment of Ref. 10,13 c an be taken into account by considering the order parameter "transverse susceptibility" $\chi^{x x}(T)$ which is related to the "transverse" pair correlation function averaged over the whole system. Although this quantity is not directly related to the magnetic susceptibility it measures the phase correlations in the whole system 18 . We have calculated this quantity as a function of temperature in the non critical region using a two spin cell approximation and the result is indicated in Fig. 2. We note that, at the critical line $\chi^{x x}(T)$ diverges as expected. An important feature to be noted is the existence of an inflexion point of the transverse susceptibility at $T=T^{*}$ which changes with $E / U$. The points in which it occur defines a line in the non-critical region of the phase diagram which is shown in Fig. 3. As expected from general scaling arguments2 
this crossover line $T^{*}(E / U)$ has the same exponential dependence found for the critical line for $(E / U)>(E / U)_{c}$. The physical meaning of the crossover line becomes clear if we recall the competition between charge and phase fluctuations which gives rise to the $T=0$ phase transition. For $T<T^{*}$ the system is in a region of rather well defined charge states loosing

phase coherence. As the temperature is increased for $(E / U)<(E / U)_{c}$ it enters a regime of strong charge fluctuations allowing for an enhancement of phase coherence which above the threshold value $(E / U)_{c}$ leads to genuine long range phase coherence. Although the crossover line shown in Fig. 3 suffers from the deficiencies inherent to mean-field like calculations there are two points worth stressing: i) anomalies in physical quantities, like minima in the resistivity, in the non-critical region of the phase diagram ,i.e., for $(E / U)<(E / U)_{c}$ , are expected to occur along the crossover line. ii) this crossover line is governed by the same exponent of the critical line as a consequence of scaling.

The quantum to classical crossover in granular superconductors has also been studied by Doniach 1 but we would like to emphasize the difference between his approach and ours. While he is studying the crossover in the critical region of the phase diagram above the transition line we are stressing effects which occur for $(E / U)<(E / U)_{c}$ that is the non-critical region of the phase diagram. This region sometimes is more amenable to experimental observation particularly in the case where critical behavior is accompanied by extreme critical slowing down which takes the system out of equilibrium close to the critical line包. Notice that since by varying the pressure in a granular material one can in principle alter the ratio $(E / U)$ then pressure measurements, under the assumption that nothing else is changing, would allow to trace the crossover line by accompanying how anomalies in the physical quantities shift with applied pressure目.

\section{CHARGE ORDERING}

We have concentrated so far on the effects of phase fluctuations treating the intergrain coupling $\mathrm{V}$ as a small parameter. Let us consider now how a larger $\mathrm{V}$ will affect the super- 
conduting transition. For this purpose we shall neglect the Josephson coupling for a while taking $E=0$. Using a spin language it is clear that while $U$ tries to stablish a singlet (neutral) ground state, the interaction $\mathrm{V}$ favors the existence of local moments ( charge disbalance ) to take advantage of the lowering of energy due to long range antiferromagnetic order ( charge instability ). For the system of superconducting grains this competition gives rise at $T=0$ to a phase transition associated with the appearance of an insulating charge ordered state for large V. In fact, the possibility of an antiferromagnetic ordering of charges has been considered sometime ago by Fazekas 1 who showed that this instability occurs for $V / U>v_{c}$, where $v_{c}=1 / c$, within mean field.

To study the effects of $V / U>v_{c}$ on the phase diagram of Fig. (1) we need to introduce two order parameters: the transverse magnetization $m_{x y}=<S^{x}>$, which describes the long range phase coherence and the staggered magnetization $m_{z}=<S^{z}>$, which represents the antiferromagnetic charge ordering. Within a mean field (one site cell) approximation this amounts to replace Eq. (2) by

$$
H=2 U\left[S^{z}\right]^{2}-4 V c m_{z} S^{z}-\frac{E}{8} c m_{x y}\left(S^{+}+S^{-}\right)
$$

and the phase boundary is obtained as usual from the solution of the self consistent equations

$$
\begin{aligned}
& m_{z}=<S^{z}> \\
& m_{x y}=<S^{+}+S^{-}>
\end{aligned}
$$

We have solved these equations numerically and obtained a phase diagram as indicated in Fig. 4. The important features to be noted are the first order nature of the superconducting transition at low temperatures, which appear as a discontinuity in the staggered order parameter $\left\langle S^{z}>\right.$, and the existence of three thermodynamically different phases. The first order character of the superconductor insulator transition at $T=0$, which we find here for large short range Coulomb repulsion, i.e. $V / U>v_{c}$, is in fact quite similar to the result of Fisher and Grinsteine for long range Coulomb interactions where this transition results second or first order depending on the parameters. Even more interesting is the topology of 
the phase diagram of Fig. 4, with superconducting, charge ordering and normal phases27. If we take into account the temperature dependence of the Josephson tunneling amplitude $E$ between the grains, which is a decreasing function of temperature 2 , we find that this topology allows for the possibility that a granular material in the $V / U>v_{c}$ regime can become a charge ordered insulator before becoming superconducting for decreasing temperature as the system moves through the path indicated in Fig. 4. Since in the charge ordered phase the system should be an insulator, the experimental signature of this effect could show up as non monotonic behavior of the resistivity with decreasing temperature which would reach a maximum just before becoming superconducting. In fact, an anomalous peak in the resistivity of some granular systems has already been observed just before the superconducting transition 17. Note that only for a restricted range of parameters near the tricritical point in Fig 4 would this effect be expected which then is consistent with the rather unusual observation of this phenomena. Of course, a satisfactory comparison of this result with experiment would require incorporating into the model several relevant complications as for example disorder 29 and dissipation 30 . We expect, however, that carefully prepared materials could show some signature of this effect.

\section{CONCLUSIONS}

We have studied a pseudospin one model of granular superconductors that takes into account the competition between charge fluctuations and phase locking in these materials. The phase diagram for the phase locking transition has been obtained within a mean field renormalization group and compared with previous calculations. No reentrant behavior has been found within this method in agreement with recent studies. We have also shown the existence of a crossover line in the non-critical region of the phase diagram and along which we expect to find anomalies in the transport and thermodynamic properties. This line could in principle be accessed experimentally by applying external pressure in the system. Finally we considered the effects of charge ordering in the phase diagram within a mean approxi- 
mation and shown that it leads to three different phases and to first order transitions at low temperatures. We have found that for a range of intergrain and Josephson coupling interactions the granular superconductor may enter an insulating charge ordered phase before becoming superconducting with decreasing temperature. We suggested that this could lead to a non monotonic behavior of the resistivity with decreasing temperature with a maximum in the resistance of the material just before becoming superconducting. This could in principle be observed experimentaly as similar anomalous peaks have already been observed in some granular superconductors.

\section{ACKNOWLEDGMENTS}

We thank R. Kishore for helpfull discussons. This work was supported in part by Conselho Nacional de Desenvolvimento Científico e Tecnológico (CNPq) (M.A.C. and E.G.). M.A.C. also acknowledges the CNPq-RHAE program for supporting a visit to the Instituto Nacional de Pesquisas Espaciais (INPE) where part of this work was done. He also thanks the members of the research group at INPE for their kind hospitality. 


\section{REFERENCES}

${ }^{1}$ G. Deutscher and D.A. Müller, Phys. Rev. Lett. 59, 1745 (1987).

${ }^{2}$ J. Rosemblatt, Rev. Appl. Phys. 9, 217 (1974).

${ }^{3}$ H.A. Borges and M.A. Continentino, Solid St. Comm. 80, 197 (1991).

${ }^{4}$ B. Abeles, Phys. Rev. B 15, 2828 (1977).

${ }^{5}$ K.B. Efetov, Sov. Phys. JETP 51, 1015 (1980).

${ }^{6}$ E. Simánek, Solid State Commun. 31, 419 (1979).

${ }^{7}$ E. Simánek, Inhomogeneous Superconductors, Troisième Cycle de la Physque, Swisse Romande(1981), unpublished.

${ }^{8}$ E. Simánek, Phys. Rev. B 23, 5762 (1982).

${ }^{9}$ P. Fazekas, Z. Phs. B45, 215 (1981).

${ }^{10}$ P. Fazekas, B. Muhlschlegel and M. Schroter, Z. Phys. B 57,193 (1984).

${ }^{11}$ S. Doniach, Phys. Rev. B 24, 5063 (1981).

${ }^{12}$ S. Doniach, in Percolation, Localization and Superconductivity, Edited by A. M. Goldman and S. A. Wolf (Plenum, New York, 1984), p. 401.

${ }^{13}$ R. Fazio and G. Giaquinta, Phys. Rev. B 34, 4909 (1986).

${ }^{14}$ R.S. Fishman and D. Stroud, Phys. Rev. B 37, 1499 (1988).

${ }^{15}$ R.S. Fishman, Phys. Rev. Lett. 63, 89 (1989).

${ }^{16}$ M.V. Simkin, Phys. Rev. B 44, 7074 (1991).

${ }^{17}$ H. Micklitz, University of Koln, preprint.

${ }^{18}$ C. Lebeau, A. Raboutou, P. Peyral and J. Rosemblatt, Physica B 152, 100 (1988). 
19 J.O. Indekeu, A. Maritan and A. Stella, J. Phy. A 15, L291 (1982).

20 J.A. Plascak, J. Phys. A 17, L597 (1984).

${ }^{21}$ M.A. Continentino and S. Coutinho, JMMM, to published.

22 M.L. Lyra and S. Coutinho, Physica A 155, 232 (1989).

${ }^{23}$ N.D. Mermin and H. Wagner, Phys. Rev. Lett. 17, 1133 (1966).

${ }^{24}$ P. Pfeuty, D. Jasnow and M.E. Fisher, Phys. Rev. B 102088 (1974).

${ }^{25}$ A.P. Malozemoff, S.E. Bardes and P. Barbara, Phys. Rev. Lett. 51, 1704 (1983).

${ }^{26}$ M.P.A. Fisher and G. Grinstein, Phys. Rev. Lett.60, 208 (1988).

${ }^{27}$ Similar topology of the phase diagram has been discussed in Ref. 12 in two dimensions for a model with negative $V$ where the charge ordering transition is replaced by a KosterlitzThouless metal-insulator transition. Long-range Coulomb interactions can also lead to similar transitions in two dimensions as discussed by J.E. Mooij et al, Phys. Rev. Lett. 65, $645(1990)$.

${ }^{28}$ V. Ambegaokar, A. Baratoff, Phys. Rev. Lett. 10, 486 (1963).

29 J.M. Kosterlitz and E. Granato, Physica B 152, 152 (1988).

${ }^{30}$ S. Chakravarty, G. Ingold, S. Kivelson and A. Luther, Phys. Rev. Lett. 56, 2303 (1986). 


\section{FIGURES}

FIG. 1. Critical temperature $T_{c}$ for the phase-locking transition as obtained by the mean field renormalization group (MFRG) and a self consistent two site cell (Oguchi) approximation.

FIG. 2. Order parameter transverse susceptibility as function of temperature for a value of $E / U$ in the insulating phase of Fig. 1.

FIG. 3. Schematic phase diagram showing the location of the quantum to classical crossover as determined from the transverse susceptibility.

FIG. 4. Phase diagram obtained by mean field approximation for $V / U=0.18$ and $c=6$. The dot-dashed line indicates first order transitions and the dashed line a path described by a sample with a temperature dependent Josephson coupling $E(T)$. 